\title{
GENERALIZED SZEGÖ THEOREMS AND ASYMPTOTICS OF CUMULANTS BY GRAPHICAL METHODS
}

\author{
FLORIN AVRAM
}

\begin{abstract}
We obtain some general asymptotics results about a class of deterministic sums called "sums with dependent indices," which generalize a classical theorem of Szegö. The above type of sums is encountered when establishing convergence to the Gaussian distribution of sums of Wick products by the method of cumulants. Our asymptotic results reduce in this situation the proof of the central limit theorem to the study of the connectivity of a family of associated graphs.
\end{abstract}

\section{INTRODUCTION}

The origins of this work are in a series of papers: Breuer and Major [BM], Giraitis and Surgailis [G1] and especially Fox and Taqqu [F2], in which the authors established convergence to the normal of certain sums by studying the asymptotic behavior of their cumulants. In Fox and Taqqu [F2], an important tool used was "the power counting conditions" (used by physicists in quantum field theory), which ensure the convergence of integrals of the form

$$
\int_{[0,1]^{C}} \frac{d y_{1} \cdots d y_{C}}{x_{1}^{z_{1}} \cdots x_{E}^{z_{E}}},
$$

where $x_{e}, e=1, \ldots, E$, are linear combinations with integer coefficients of $y_{1}, \ldots, y_{C}$, taken modulo 1 , and $z_{e}$ are positive numbers. Let

$$
\left(x_{1}, \ldots, x_{E}\right)=\left(y_{1}, \ldots, y_{C}\right) M,
$$

where $M$ is the $C \times E$ matrix of the dependence. It was known that the integral (1.1) converges if for any set of columns $A$ of the matrix $M$, the power counting conditions

$$
\sum_{e \in A} z_{e} \leq \operatorname{rank}(A), \quad \forall A \subset\{1, \ldots, E\}
$$

are satisfied.

In this paper, and the paper [AB], we improve on the previous work in two respects.

Received by the editors February 9, 1989 and, in revised form, January 9, 1990.

1980 Mathematics Subject Classification (1985 Revision). Primary 60F05, 60C05.

Key words and phrases. Power counting conditions, generalized Hölder inequality, Szegö theorem, bond matroid, diagram formulae.

Work partly supported by the U.S. Army Research Office through the Mathematical Sciences Institute of Cornell University. 
(a) We showed in [AB] that under the conditions (P.C.), a Hölder type inequality holds for integrals of the form (1.1). This in turns leads here to some interesting asymptotic results related to a theorem of Szegö, presented in $\S$ I, A and $B$.

(b) We note the existence in matroid theory of various formulae for the function $r(A)$ in the R.H.S. of (1.2), which simplify the analysis of the conditions (P.C.). An example is formula (1.13) in $\S \mathrm{I}, \mathrm{C}$, applicable when the matroid is induced by a graph.

Using the method of cumulants, in conjunction with the previously mentioned tools, we establish in §II quite complicated central limit theorems for sums of Wick products of Gaussian random variables.

The proofs of the results in $\S I$ are given in $\S I I I$.

In the companion paper [AF], we establish similar results for non-Gaussian random variables, unfortunately under an assumption whose applicability is difficult to check.

\section{Generalized Szegö THEOREMS}

A. Sums with dependent indices. Let $f^{(e)}(x), e=1, \ldots, E$, be $E$ functions on the torus $[0,1]$, extended periodically to the whole line, with $f^{(e)} \in L_{p_{e}}$, $e=1, \ldots, E$. Let $\hat{f}_{k}^{(e)}$ denotes the Fourier coefficients of $f^{(e)}(x)$, i.e.:

$$
\hat{f}_{k}^{(e)}=\int_{0}^{1} e^{2 \pi i k x} f^{(e)}(x) d x, \quad e=1, \ldots, E .
$$

Definition. A sum with dependent indices is a sum of the form

$$
S_{n}=S_{n}\left(M, f^{(e)}, e=1, \ldots, E\right)=\sum_{j_{1}, \ldots, j_{V}=1}^{n} \hat{f}_{i_{1}}^{(1)} \cdots \hat{f}_{i_{E}}^{(E)}
$$

where $M$ is a $V \times E$ matrix with integer entries and.

$$
\left(i_{1}, \ldots, i_{E}\right)=\left(j_{1}, \ldots, j_{V}\right) M \text {. }
$$

The nullity of the map $x M$ will be denoted by $\mu(\mu=V-\operatorname{rank}(M))$. Throughout the paper, we make the assumption

$$
\begin{aligned}
& \text { for any row } r \text { in } M, \operatorname{rank}(M)=\operatorname{rank}(M \backslash r) . \\
& \text { Assumption (A) implies } \mu \geq 1 .
\end{aligned}
$$

Let now $M^{*}$ be an integer matrix representing the matroid dual to the matroid of $M$, i.e., a matrix having the same number of columns $E$ as $M$, and such that the rows of $M^{*}$ form a basis in the subspace orthogonal to the rows of $M$. The number of rows $C$ of $M^{*}$ is thus $C=E-(V-\mu)$.

(Our notation is inspired by the important particular case in which $M$ is the incidence matrix of a graph $G$ with $V$ vertices and $E$ edges, $C$ is the maximal number of independent cycles in $G, \mu$ is the number of connected components of $G$, and the formula above is Euler's formula.)

For any set $A \subset\{1, \ldots, E\}$ let $r(A) \quad\left(r^{*}(A)\right)$ denote the rank of the set of columns of $M\left(M^{*}\right)$ indexed by $A$. Let also $z_{e}=\left(p_{e}\right)^{-1}$ be the reciprocals of the integrability factors $p_{e}$. It turns out that the order of magnitude of the sums $S_{n}(M)$, denoted by $\alpha_{M}(\mathbf{z})$, depends on $\mathbf{z}=\left(z_{1}, \ldots, z_{E}\right)$ and on the rank function $r^{*}$. 
Theorem 1. If the matrix $M$ satisfies assumption (A), then

$$
\left|S_{n}\left(M, f^{(e)}, e=1, \ldots, E\right)\right| \leq c_{M} n^{\alpha_{M}(\mathbf{z})} \prod_{e=1}^{E}\left\|f^{(e)}\right\|_{p_{e}},
$$

where $c_{M}$ is a constant, and

$$
\alpha_{M}(\mathbf{z})=\mu+\max _{A \subset\{1, \ldots, E\}}\left[\sum_{e \in A} z_{e}-r^{*}(A)\right] .
$$

B. The generalized Szegö theorem. It follows from (1.5) that

$$
\mu \leq \alpha_{M}(\mathbf{z}) \leq V
$$

and $\alpha_{M}(\mathbf{z})=\mu$ iff $\sum_{A} z_{e} \leq r^{*}(A), \forall A \subset\{1, \ldots, E\}$.

It turns out that when $\alpha_{M}(\mathbf{z})=\mu$, the inequality (1.4) is in fact tight, and a Szegö type theorem holds for the sums $S_{n}(M)$. Let

$$
I=I\left(M^{*}, f^{(e)}, e=1, \ldots, E\right):=\int_{[0,1]^{c}} \prod_{e=1}^{E} f^{(e)}\left(x_{e}\right) \prod_{c=1}^{C} d y_{c},
$$

where $\left(x_{1}, \ldots, x_{e}\right)=\left(y_{1}, \ldots, y_{c}\right) M^{*}$, each $x_{e}$ being reduced modulo $[0,1]$.

Theorem 2. Let $M$ satisfy assumption (A), and let $f^{(e)}(x) \in L_{p_{e}}, e=1, \ldots, E$, where $L_{p}$ denotes the closure of trigonometric polynomials in the $L_{p}$ sense, i.e.:

$$
L_{p}=\left\{\begin{aligned}
L_{p} & \text { if } p \neq \infty \\
C & \text { if } p=\infty
\end{aligned}\right.
$$

If $\alpha_{M}(\bar{z})=\mu$, or, equivalently,

$$
\sum_{e \in A} z_{e} \leq r^{*}(A), \quad \forall A \subset\{1, \ldots, E\}
$$

then,

$$
\frac{1}{n^{\mu}} S_{n}(M) \rightarrow c_{M} I\left(M^{*}\right)
$$

(the constant $c_{M}$ is defined in 2.8).

Note. The conditions (1.9) imply that the R.H.S. of (1.10) is well defined and a continuous multilinear functional, by the "generalized Hölder inequality" (see Theorem 1 of $[\mathrm{AB}])$.

The method used in proving Theorem 2 yields also

Corollary 1. If $\alpha_{M} \geq \mu$ and $f^{(e)} \in t_{p_{e}}$, for $e=1, \ldots, E$, then

$$
S_{m}(M)=o\left(n^{\alpha_{M}}\right) \text {. }
$$

C. Graph sums. An interesting special case of a sum with dependent indices is that in which the dependency matrix $M$ is associated with a graph. Let $G=(\mathscr{V}, \mathscr{E})$ be a directed graph with $V$ vertices, $E$ edges, and $\mu$ components. For any $v \in \mathscr{V}$ and $e \in \mathscr{E}$, let

$$
M_{v, e}=\left\{\begin{aligned}
0 & \text { if } v \notin e \\
1 & \text { if } v \text { is the end point of } e \\
-1 & \text { if } v \text { is the start point of } e
\end{aligned}\right.
$$


In this case, we will denote the sums (1.3) by $S_{n}(G)$, and call them "graph sums." Since this $M$ represents the cycle matroid of the graph (see Bixby [B, p. 350], or Welsh [W, pp. 171-172]) its dual is the bond matroid. Let $c(A)$ denote the number of components in the graph $(\mathscr{V}, A)$ it is known that the rank function of the bond matroid is given by

$$
r^{*}(A)=|A|-c(\mathscr{E} \backslash A)+\mu,
$$

where $c(\mathscr{E} \backslash A)$ denotes the number of components left in $G$ after the edges indexed by $A$ have been removed. (This formula follows for example from the formulas

$$
r^{*}(A)=|A|-r(M)+r(M \backslash A)
$$

and $r(A)=V-c(A)$, of $[\mathrm{W}$, p. $35,(2.1 .5)$ and p. 29, (1.10.5)].)

Formula (1.5) becomes then in this case

$$
\alpha_{G}=\max _{A \subset\{1, \ldots, E\}}\left[c(\mathscr{E} \backslash A)-\sum_{e \in A}\left(1-z_{e}\right)\right] .
$$

It is basically the use of this formula that makes the deriving of our C.L.T.'s in the next section simpler than the similar results of previous authors. One can think of formula (1.13) as of a "game of breaking the graph:" removing an edge $e$ "costs" $1-z_{e}$, and breaking a new component brings a "benefit" of 1 ; $\alpha_{G}$ is then the maximal profit possible.

We point out now the particular form Theorems 1,2 and Corollary 1 take in the case of graph sums.

Theorem 3. Let $S_{n}(G)$ be the graph sums associated to a graph $G$, let $\mu$ denote the number of components of the graph, let $C=E-(V-\mu)$ denote the maximal number of independent cycles of $G$ and let $M^{*}$ be a $C \times E$ matrix defined as follows: select a maximal set $\mathscr{C}$ of $C$ independent cycles in $G$, assign them arbitrary orientations and let, for $c=1, \ldots, C$ and $e=1, \ldots, E$,

$$
\left(M^{*}\right)_{c, e}=\left\{\begin{aligned}
0 & \text { if } e \neq c, \\
1 & \text { if } e \in c, \text { and their orientations coincide, } \\
-1 & \text { if } e \in c, \text { and they have opposite orientations. }
\end{aligned}\right.
$$

Then

(a) $\left|S_{n}(G)\right| \leq c_{G} n^{\alpha_{G}} \prod_{e=1}^{E}\left\|f^{(e)}\right\|_{p_{e}}$.

(b) If, moreover, $f^{(e)} \in L_{p_{e}}$, for $e=1, \ldots, E$, and $\forall A \subset \mathscr{E}$,

$$
\sum_{e \in A}\left(1-z_{e}\right) \geq c(G \backslash A)-\mu
$$

we have

$$
\frac{1}{n^{\mu}} S_{n}(G) \rightarrow I(G):=\int_{[0,1]^{C}} \prod_{e=1}^{E} f^{(e)}\left(x_{e}\right) \prod_{c=1}^{C} d y_{c},
$$

where $\mathbf{x}=\mathbf{y} M^{*}$.

(c) If $\alpha_{G}>\mu$, then $S_{n}(G)=o\left(n^{\alpha_{G}}\right)$.

Note. Theorem 3(b) was first obtained in $[\mathrm{AB}]$. Two particular cases of it were already well known: (a) when the graph $G$ is a cycle, and $f^{(1)}=\cdots=f^{(E)}$, 
Theorem 3(b) reduces to a well-known result of Szegö (improved in [A]) on the trace of a product of Toeplitz matrices. (b) When $E=2$, Theorem 3(b) reduces to the classical Parseval relation (see Katznelson [K, p. 35]).

\section{THE CENTRAL LIMIT THEOREM}

Theorem 3 is a convenient tool for establishing central limit theorems by the method of cumulants.

Corollary 2. Let $T_{n}$ be a sequence of zero mean random variables, with cumulants of all orders, for which

$$
\operatorname{cum}_{s}\left(T_{n}\right)=\sum_{G \in \mathscr{g}_{s}} S_{n}(G),
$$

where $S_{n}(G)$ are graph sums, and the summation runs over all $G$ in a certain family of connected graphs, $g_{s}$. Then, if

$$
\alpha_{G} \leq s / 2, \quad \forall G \in g_{s},
$$

the central limit theorem $T_{n} / \sigma \sqrt{n} \stackrel{d}{\longrightarrow} N(0,1)$ holds, with $\sigma^{2}=\sum_{G \in g_{2}} I(G)$.

Proof. Here, $\mu=1, \forall G \in g_{s}$. Note now that $\operatorname{cum}_{s}\left(T_{n} / \sigma \sqrt{n}\right) \underset{n \rightarrow \infty}{\longrightarrow} \delta_{2}(s)$, by applying Theorem 3(b) when $s=2$, and 3(c) when $s \geq 3$.

Corollary 2 may reduce the establishing of very complicated C.L.T's to some simple "graph breaking" problems. We consider now two such specific situations.

Let $X_{i}$ be a $\phi$ mean, stationary Gaussian sequence, with $E X_{\phi}^{2}=1$, and spectral function $f(x)$ (i.e., $\left.E X_{\phi} X_{k}=\int e^{2 \pi i k x} f(x) d x\right)$, with $f(x) \in \mathrm{L}_{p_{1}}$, let $a(x) \in \mathrm{L}_{p_{2}}$, with $a(x)$ even and let $\hat{a}_{k}$ denote its Fourier coefficients. Let $z_{i}:=\left(p_{i}\right)^{-1}$, for $i=1,2$.

We will present conditions on the $z_{i}$ which imply the C.L.T. For the following two types of sums:

$$
\begin{gathered}
T_{n}=\sum_{j=1}^{n}: X_{j}^{(m)}: \\
T_{n}=\sum_{j, k=1} a_{j-k}: X_{j}^{(m)}, X_{k}^{(l)}:
\end{gathered}
$$

where $: X_{j}^{(m)}:,: X_{j}^{(m)}, X_{k}^{(l)}$ : denote respectively the $m$ th Wick power of $X_{j}$ and the Wick product of $X_{j} m$ times and $X_{k} l$ times. for a definition of the Wick products, see [G2]; below, however, we will need only to use the fact that the cumulants of Wick products can be conveniently expanded by means of the diagram formula (see [G2, Theorem 4(IV)]).

Theorem 4. (a) If $T_{n}$ is defined by (2.3a), and $z_{1} \leq 1-1 / m$, then

$$
T_{n} / \sqrt{n} \rightarrow N\left(0, \sigma^{2}\right)
$$

with $\sigma^{2}=\sum_{G \in g_{2}} I(G)$.

Here, $g_{s}$ is the family of connected undirected graphs with $s$ vertices each having degree $m$. 


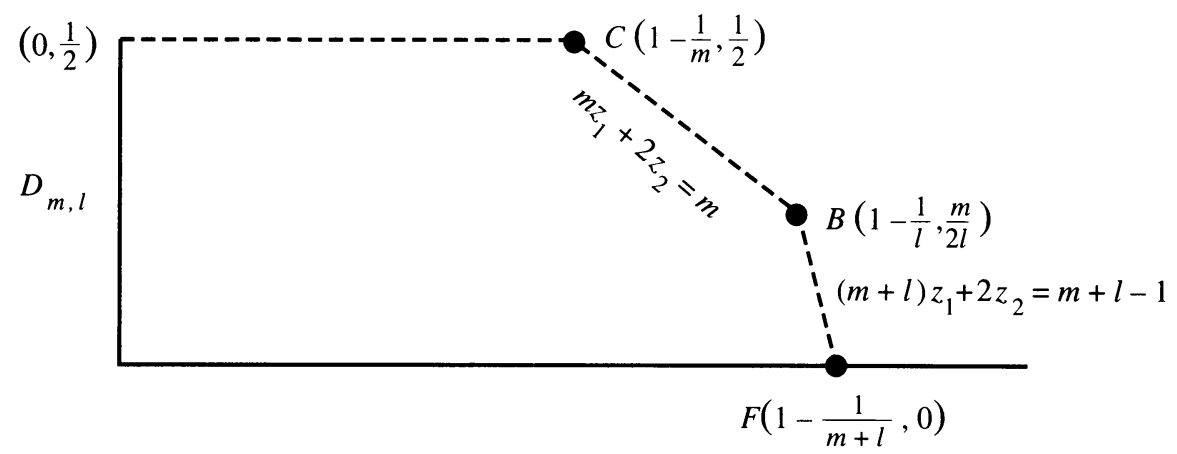

Figure 1

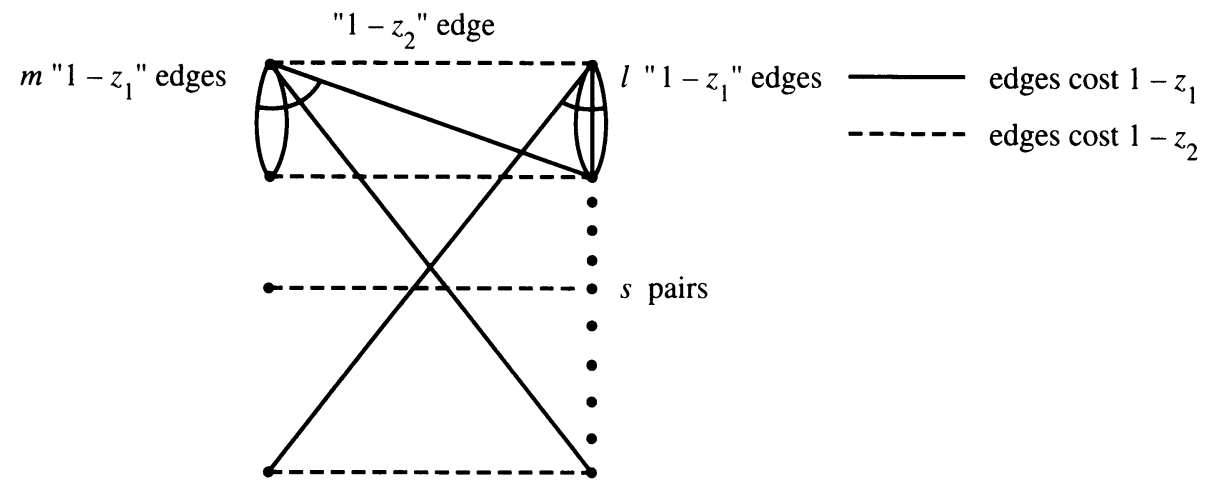

FIGURE 2

(b) If $T_{n}$ is defined by (2.3b), $2 \leq m \leq l$, and $\left(z_{1}, z_{2}\right) \in D_{m, l}$ where

(2.4) $D_{m, l}=\left\{\left(z_{1}, z_{2}\right) \in[0,1]^{2}: z_{2} \leq 1 / 2\right.$,

$$
\left.(m+l) z_{1}+2 z_{2} \leq m+l-1, m z_{1}+2 z_{2} \leq m\right\},
$$

then $T_{n} / \sqrt{n} \rightarrow N\left(0, \sigma^{2}\right)$, with $\sigma^{2}=\sum_{G \in g_{2}} I(G)$ (see Figure 1). Here, $g_{s}$ is the family of all connected undirected graphs formed of $s$ "horizontal" pairs of vertices, each horizontal pair being connected by an edge with "price" $1-z_{2}$, and $(m+1) s / 2$ "nonhorizontal" edges (i.e., which cannot connect two left vertices of the same pair), with price $1-z_{1}$, and arranged such that the left vertices of each pair have all degree $m+1$, and the right vertices have degree $l+1$ (see Figure 2).

Proof. Both (a) and (b) follow from Corollary 2.

One has to show first that $(2.1)$ holds, where $g_{s}$ is the corresponding family of graphs. We show now this in case (a) (case (b) requires only minor modifications). By the multilinearity of cumulants,

$$
\begin{aligned}
\operatorname{cum}_{s}\left(T_{n}\right) & =\operatorname{cum}\left(\sum_{j_{1}=1}^{n}: X_{j_{1}}^{(m)}:, \ldots, \sum_{j_{s}=1}^{n}: X_{j_{s}}^{(m)}:\right) \\
& =\sum_{\mathbf{j} \in\{1, \ldots, n\}} \operatorname{cum}_{s}\left(: X_{j_{1}}^{(m)}:, \ldots, X_{j_{s}}^{(m)}\right) .
\end{aligned}
$$


By the diagram formula (see Theorem 4(IV) of [G2])

$$
\begin{aligned}
\operatorname{cum} & \left(: X_{j_{1}}^{(m)}:, \ldots,: X_{j_{s}}^{(m)}\right) \\
& =\sum_{P=\left\{\left(j_{k_{1}}, j_{l_{1}}\right), \ldots,\left(j_{k_{m s / 2}}, j_{l_{m s / 2}}\right)\right\} \in P} r_{j_{k_{1}}-j_{l_{1}}} \cdots r_{j_{k_{m s / 2}}-j_{l_{m s / 2}}}
\end{aligned}
$$

where $\mathscr{P}$ is the set of partitions in pairs of the table

$$
\begin{array}{ll}
j_{1}, \ldots, j_{1} \quad(m \text { times }), \\
\vdots \\
j_{s}, \ldots, j_{s} \quad(m \text { times }),
\end{array}
$$

such that each pair connects two distinct rows, and no subset of pairs has as union a subset of rows strictly included in $\{1, \ldots, s\}$.

Note now that the set of partitions $\mathscr{P}$ can be put in a 1-to-1 correspondence with the set of connected unoriented graphs $\mathscr{G}_{s}$ with $s$ vertices of degree $m$ (one for each index $j_{k}, k=1, \ldots, s$ ), by representing each partition pair $\left(j_{k}, j_{l}\right)$ as an edge between the vertices associated to $j_{k}, j_{l}$. Finally, note that summing $\mathbf{j}$ in each term in the R.H.S. of (2.5) corresponding to fixed $P$ yields a sum of the form (1.3), with the matrix $M$ being the incidence matrix of the graph $G$, arbitrarily oriented (since $r_{k}$ (and $\hat{a}_{k}$ in case (b)) is an even sequence, $S_{n}(G)$ does not depend on the orientation of the edges).

To end the proof, it remains now only to check that (2.2) holds. In case (b), this is done in Lemma 1. We proceed now to show that (2.2) holds in case (a). Since the function $\alpha_{G}\left(z_{1}\right)$ is increasing in $z_{1}$, it will be enough to consider the "worst" case $z_{1}=1-1 / m$.

Let the "profit" of a set of edges $A$ be

$$
p(A)=c(\mathscr{E} \backslash A)-\sum_{e \in A}\left(1-z_{e}\right) .
$$

To find $\alpha_{G}=\max p(A)$, it is enough to find a set $A$ which achieves the maximum profit, and which is also maximal with respect to inclusion. We will call such a set $A$ a maximal optimal breaking, M.O.B.

We will show now that when the cost of breaking an edge, $1-z_{1}$, equals $1 / m$, the "total breaking" is the unique M.O.B. Indeed, suppose that after applying a M.O.B., one vertex would be still connected to some others. Cut now all the edges around this vertex. At a cost of no more than $m \times 1 / m$, we increase the profit by the least 1 , contradicting thus that we had a M.O.B.

Finally, note that the profit of the M.O.B. at $z_{1}=1-1 / m$ is $p(\mathscr{E})=$ $s-m s / 2,1 / m=s / 2$, and thus

$$
\alpha_{G}(1-1 / m)=p(\mathscr{E})=s / 2, \quad \forall G \in \mathscr{G}_{s},
$$

establishing thereby (2.2) and Theorem 4 in the case (a).

Note. Theorem 4(a) was obtained by Breuer and Major [BM]. We included it here to illustrate the fact that our method reduces the quite involved initial proof to the very simple "graph breaking" problem above. The "graph breaking" problem in the more complicated case (b) can be also handled in a similar way. 
Lemma 1. Let $\mathscr{G}_{s}$ be the family of graphs of Theorem 4(b). Then $\forall\left(z_{1}, z_{2}\right) \in$ $\mathscr{D}_{m, l}$ (defined in (2.4)), and $\forall G \in \mathscr{G}_{s}, s \geq 2$, we have

$$
\alpha_{G}\left(z_{1}, z_{2}\right) \leq s / 2 \text {. }
$$

Proof. Since $\alpha_{G}(\mathbf{z})$ is convex and increasing in $\mathbf{z}$, it is enough to establish (2.6) for the 3 extremal points of $\mathscr{D}_{m, 1}$ :

$$
F(1-1 / m+1,0), \quad B(1-1 / l, m / 21), \quad C(1-1 / m, 1 / 2) .
$$

For the point $F$, one notes that since removing $z_{2}$ edges costs 1 , there can be no profit in removing them. This in effect fuses together each horizontal pair into a single point, of degree $m+l$, each edge having a cost of $1 /(m+1)$, and so we are back in the case of Theorem $4(a)$, and thus

$$
\alpha_{G}(F)=s-\frac{(m+1) s}{2}\left(1-z_{1}\right)=\frac{s}{2} .
$$

For the point $B$, we note first that the total breaking $A=\mathscr{E}$ achieves a profit of $s / 2$ :

$$
p(\mathscr{E})=2 s-\frac{(m+1) s}{2} \frac{1}{l}-s\left(1-\frac{m}{21}\right)=\frac{s}{2} .
$$

Next, we show that $\mathscr{E}$ is the (only) M.O.B. at this cost, i.e. there after the removal of a M.O.B. there can be no component left which is not a singleton. Indeed, note first that a nonsingleton component cannot contain only one of the vertices of a "horizontal" pair, since by further disconnecting this vertex we could increase the benefit by 1 at a cost of a most $l \frac{1}{l}$, thereby increasing the profit. Thus, the nonsingleton component has to be formed of a set of $K$ "horizontal" pairs, for some $k$. However, just like in (2.7), by totally breaking the component one would increase the profit by $k / 2-1$, and thus we cannot have $k \geq 2$. Since clearly a M.O.B. cannot leave a one pair component unbroken, it follows that the only components left can be singletons, and M.O.B. $=\mathscr{E}$. Hence, $\alpha_{G}(B)=p(\mathscr{E})=s / 2$.

For the point $C$, the M.O.B. might depend on which particular graph in $\mathscr{G}_{s, m, l}$ we break. However, by an analysis similar with that of point $B$, one can show that a M.O.B. has to break isolated all the points on the left side of the graph. Let then $c_{k}$ be the number of components of $k$ points on the right side of the graph, and $r$ the number of edges connecting two points on the right side, left after the removal of a M.O.B. We have to show

$$
\alpha_{G}(C)=s+\sum_{i=1}^{\infty} c_{i}-\left[\frac{(m+l) s}{2}-r\right] \frac{1}{m}-\frac{s}{2} \leq \frac{s}{2},
$$

or

$$
\sum_{i=1}^{\infty} c_{i} \leq \frac{s}{2}+\left[\frac{l s}{2}-r\right] \frac{1}{m}
$$

Since

$$
\frac{c_{1}}{2}+\sum_{i=2}^{\infty} c_{i} \leq \frac{\sum_{i=1}^{\infty} i c_{i}}{2}=\frac{s}{2},
$$

it is enough to show that

$$
\frac{m c_{1}}{2} \leq\left(\frac{l s}{2}-r\right)
$$


Let now $b, d$ denote the number of edges in the initial graph with both ends in the right side, and with ends on different sides, respectively. Thus, $d+2 b=l s$. Also, let $b^{\prime}=b-r$ the number of edges with both ends on the right side which have been removed by the M.O.B. We have

$$
l c_{1} \leq d+2 b^{\prime}=d+2(b-r)=l s-2 r,
$$

and thus (2.8) follows.

\section{Proofs of Results IN $§ I$}

Proof of Theorem 1. We will need the following integral representation of $S_{n}(M)$ :

$$
S_{n}(M)=\int_{[0,1]^{E}} \prod_{v=1}^{V} \Delta_{n}\left(v_{n}\right) \prod_{e=1}^{E} f^{(e)}\left(x_{e}\right) d x_{e},
$$

where $\Delta_{n}(x)=\sum_{k=1}^{n} e^{2 \pi i k x}$, and $u_{v}=\sum_{e} M_{v, e} x_{e}$ : as in Lemma 1 of [AB], (3.1) is obtained by plugging $\int e^{2 \pi i k x_{e}} f^{(e)}\left(x_{e}\right) d x_{e}$ instead of $f_{k}^{(e)}$ in (1.3).

Let us note now that

$$
\left\|\Delta_{n}\right\|_{s^{-1}} \leq k(s) n^{1-s}, \quad \forall s \in[0,1),
$$

where the constant $k(s)$ increases with $s$ and explodes at $s=1$.

To prove Theorem 1, we apply now to the integral representation (3.1) the generalized Hölder inequality (Theorem 1) of $[\mathrm{AB}]$. We get

$$
\begin{aligned}
& \left|S_{n}(M)\right| \leq \prod_{v=1}^{V}\left\|\Delta_{n}\right\|_{1 /\left(s_{v}\right)} \prod_{e=1}^{E}\left\|f^{(e)}\right\|_{p_{e}} \\
& \leq \prod_{v=1}^{V} k\left(s_{v}\right) n^{\sum_{v=1}^{V}\left(1-s_{v}\right)} \prod_{e=1}^{E}\left\|f^{(e)}\right\|_{p_{e}},
\end{aligned}
$$

$\forall s_{1}, \ldots, s_{V} \in[0,1)$ so that the (P.C.) conditions for $\left(z_{e}, e=1, \ldots, E, s_{v}\right.$, $v=1, \ldots, V)$ and $\left(x_{e}, e=1, \ldots, E, u_{v}, v=1, \ldots, V\right)$ are satisfied.

Theorem 1 is then an immediate corollary of (3.3) and of

\section{Lemma 2.}

$$
\alpha_{M}=\min \sum_{v=1}^{V}\left(1-s_{v}\right),
$$

where the minimum is taken over all $s_{1}, \ldots, s_{V} \in[0,1)$ so that $\left(z_{e}, e=\right.$ $\left.1, \ldots, E, s_{v}, v=1, \ldots, V\right)$ and $\left(x_{e}, e=1, \ldots, E, u_{v}, v=1, \ldots, V\right)$ satisfy the (P.C.) conditions. Furthermore, the minimizing $\left(s_{1}, \ldots, s_{V}\right)$ can be chosen such that $s_{i} \leq 1-1 / V, \forall i$.

Proof of Lemma 2. Consider the matrix $T$ representing the matroid $\left(x_{e}, e \in\right.$ $\left.\{1, \ldots, E\}, u_{v}, v \in\{1, \ldots, V\}\right)$ :

$$
T=\left(I_{E}, M^{t}\right),
$$


when $I_{n}$ denotes the identity matrix over $\mathbf{R}^{n}$. We have to find

$$
\begin{gathered}
\min \sum_{v=1}^{V}\left(1-s_{v}\right) \text {, under the (P.C.) constraints } \\
\left\{\begin{array}{l}
\sum s_{v} \leq r(A, B)-\sum_{e \in A} z_{e}, \quad \forall B \subset\{1, \ldots, V\}, \forall A \subset\{1, \ldots, E\}, \\
s_{v} \geq 0, v=1, \ldots, V .
\end{array}\right.
\end{gathered}
$$

Here, $r(A, B)$ denotes the rank of the corresponding columns in the matrix $T$. Letting

$$
d_{B}=\min _{A \subset\{1, \ldots, E\}}\left[r(A, B)-\sum_{e \in A} z_{e}\right],
$$

the constraints can be further written as

$$
\begin{cases}\sum_{v \in B} s_{v} \geq d_{B}, & \forall B \subset\{1, \ldots, V\}, \\ s_{v} \geq 0, & \forall v .\end{cases}
$$

Using the constraint for $\mathscr{V}:=\{1, \ldots, V\}$, we get

$$
\min \sum_{v=1}^{V}\left(1-s_{v}\right) \geq V-d_{\mathscr{V}}
$$

The proof proceeds as follows:

(a) We find a point satisfying (3.5), where the constraints $\sum_{v=1}^{V} s_{v}=d_{\mathscr{V}}$ holds, proving thereby that (3.6) holds with equality:

$$
\min \sum_{v=1}^{V}\left(1-s_{v}\right)=V-d_{\mathscr{V}}
$$

(b) We show furthermore that the point above can be chosen such that $s_{v} \leq$ $1=1 / V, \forall v$.

(c) We show that $r(A, \mathscr{V})-\mu+r^{*}(A)$, which together with (3.7) leads to the desired formula

$$
\min \sum_{v=1}^{V}\left(1-s_{v}\right)=\mu+\max _{A \subset\{1, \ldots, E\}}\left[\sum_{e \in A} z_{e}-r^{*}(A)\right] .
$$

(a) Note that the function $d_{B}$ is submodular, i.e. $d_{B_{1} \cup B_{2}}+d_{B_{1} \cap B_{2}} \leq d_{B_{1}}+d_{B_{2}}$, since the rank function $r(A, B)$ is submodular. Thus, the polytope determined by the constraints (3.5) is a polymatroid (see Welsh, 18,3, Theorem 1); for this type of polytopes, explicit formula for the vertices are available (see [W, 18, 4, Theorem 1]); namely, for any permutation $\sigma=\left(i_{1}, i_{2}, \ldots, i_{V}\right)$, the formulas $s_{i_{1}}=d_{\left\{i_{1}\right\}}, s_{i_{2}}=d_{\left\{i_{1}, i_{2}\right\}}-d_{\left\{i_{1}\right\}}, \ldots, s_{i_{V}}=d_{\left\{i_{1}, \ldots, i_{V}\right\}}-d_{\left\{i_{1}, \ldots, i_{V-1}\right\}}$ yield a vertex $s^{(\sigma)}$ of the polytope. For this vertex, $\sum_{v=1}^{V} s_{v}^{(\sigma)}=d_{\mathscr{V}}$, which establishes (3.6). 
(b) Note also that the vertex above satisfies $s_{i_{V}}^{(\sigma)}=0$, since by assumption (A),

$$
\begin{aligned}
d_{\mathscr{V}} & =\min _{A}\left[(A, \mathscr{V})-\sum_{e \in A} z_{e}\right] \\
& =\min _{A}\left[r\left(A, \mathscr{V} \backslash i_{V}\right)-\sum_{e \in A} z_{e}\right]=d_{\mathscr{V} \backslash i_{V}} .
\end{aligned}
$$

Let now $\sigma$ denote the cyclic permutation $(2,3, \ldots, V, 1)$, and consider the vertices $s^{\left(\sigma^{i}\right)}, i=1,2, \ldots, V$, where $\sigma^{i}$ is the $i$ th iteration of $\sigma$. For each of these $V$ points, $\sum_{v=1}^{V}\left(1-s_{v}^{\left(\sigma^{i}\right)}\right)=V-d_{\mathscr{V}}$ and $s_{i}^{\left(\sigma^{i}\right)}=0$.

Letting now $\mathbf{s}=(1 / V) \sum_{v=1}^{V} s^{\left(\sigma^{i}\right)}$, we obtain a point where the minimum is achieved, and with all coordinates less than $1-1 / V$.

(c) Consider the general formula $\operatorname{dim}(C \cup B)=\operatorname{dim}(C)+\operatorname{dim}\left(P_{B}\left(C^{\perp}\right)\right)$, where $C^{\perp}$ is the orthogonal complement of $C$. We apply this to $C$ $=\operatorname{span}\left\{m_{1}^{t}, \ldots, m_{V}^{t}\right\}$, where $m_{1}, \ldots, m_{V}$ are the rows of $M$, and $B=$ $\operatorname{span}\left(e_{i}, i \in A\right)$, where $e_{i}$ is the $i$ th unit vector. We get then

$$
\begin{aligned}
r(A,\{1, \ldots, V\}) & =r(\mathscr{V})+\operatorname{dim}\left(P_{\text {span }\left(e_{i}, i \in A\right)}\left(\operatorname{span}\left(m_{1}^{t}, \ldots, m_{V}^{t}\right)^{\perp}\right)\right) \\
& =V-\mu+\operatorname{dim}\left(P_{\operatorname{span}\left(e_{i}, i \in A\right)}\left(\operatorname{span}\left(m_{1}^{t}, \ldots, m_{V}^{t}\right)^{\perp}\right)\right) .
\end{aligned}
$$

The rows of $M^{*}$ form a basis for $\operatorname{span}\left(m_{1}^{t}, \ldots, m_{V}^{t}\right)^{\perp}$. A moment's thought reveals that the dimension of the projection of the row space of $M^{*}$ on the span of $e_{i}, i \in A$, is given by the rank of submatrix of $M^{*}$ consisting of the columns with indices $i \in A$, yielding thus the formula $r(A, \mathscr{V})=V-\mu+r^{*}(A)$.

Proof of Theorem 2. For a given matrix $M$, the sequence of multilinear functionals

$$
T_{n}\left(f^{(1)}, \ldots, f^{(E)}\right):=n^{-\mu} S_{n}(M)
$$

is, by Theorem 1 , uniformly bounded on $L_{p_{1}} \times \cdots \times L_{p_{E}}$. In Lemma 3 below we prove that $(1.10)$ holds when $f^{(e)}, e=1, \ldots, E$, are the trigonometric functions $f^{(e)}(x)=e^{2 \pi i k_{e} x}$. By multilinearity this can be extended to the case of trigonometric polynomials. These being dense in $L_{p}$ we can use the uniform boundedness of $T_{n}$ to obtain (1.10) for all $L_{p}$ functions.

Lemma 3. Let $f^{(e)}(x)=e^{2 \pi i k_{e} x}, e=1, \ldots, E$, where $\mathbf{k}=\left(k_{1}, \ldots, k_{E}\right)$ is a vector of integers. Let $S_{n}^{(\mathbf{k})}(M), I^{(\mathbf{k})}\left(M^{*}\right)$ denote $S_{n}\left(M, f^{(e)}, e=1, \ldots, E\right)$ and $I\left(M^{*}, f^{(e)}, e=1, \ldots, E\right)$ in this case. Then, $\forall \mathbf{k} \in \mathbf{Z}$, we have

$$
\lim _{n \rightarrow \infty} \frac{1}{n^{\mu}} S_{n}^{(\mathbf{k})}(M)=c_{M} I^{(\mathbf{k})}\left(M^{*}\right),
$$

where the constant $c_{M}$ is defined in (3.11).

Proof. Note that the L.H.S. of (3.8) is

$$
\begin{aligned}
S_{n}^{(\mathbf{k})}(M) & :=\sum_{j_{1}, \ldots, j_{v}=1}^{n} \delta_{k_{1}}\left(i_{1}\right) \cdots \delta_{k_{E}}\left(i_{E}\right) \\
& =\operatorname{card}\left\{\mathbf{j} \in\{1, \ldots, n\}^{V}: \mathbf{j} M=\mathbf{k}\right\},
\end{aligned}
$$


and the R.H.S. of (3.8) is

$$
\begin{aligned}
I^{(\mathbf{k})}\left(M^{*}\right) & :=\int_{[0,1]^{C}} e^{2 \pi i \mathbf{x} \mathbf{k}^{t} d \mathbf{y}} \\
& =\int_{[0,1]^{C}} e_{\left\{M^{*} \mathbf{k}^{t}=\phi^{t}\right\}}^{2 \pi i \mathbf{y}=1}=1_{\{\mathbf{k} \in R(M)\}},
\end{aligned}
$$

where $R(M)$ denotes the subspace generated by the rows of $M$. (Note also at this point that the R.H.S. of (3.8) is independent of the particular choice of the matrix chosen to represent $M^{*}$.)

We consider now three cases:

(a) if $\mathbf{k} \notin R(M)$, then we get 0 in both (3.9) and (3.10).

(b) The case $\mathbf{k}=\mathbf{0}$. Note that since $M$ is an integer matrix, the set $K$ of all integer solutions of $\mathbf{j} M=\mathbf{0}$ is a module of rank $\mu$, contained in the $\mu$-dimensional space $N=\{\mathbf{u}: \mathbf{u} M=\mathbf{0}\}$. We want to determine

$$
S_{n}^{(\mathbf{0})}(M)=\operatorname{card}\left(K \cap[0, n]^{V}\right)=\operatorname{card}\left(\frac{1}{n} K \cap[0,1]^{V}\right) \text {. }
$$

Let $m_{b}$ denote the determinant of a basis of $K$ (= Lebesgue measure on $N$ of a basic cell of $K$ ), let $m_{1}$, denote the Lebesgue measure on $N$ of $N \cap[0,1]^{V}$, and let

$$
c_{M}=m_{1} / m_{b} .
$$

An elementary consideration (tantamount to the definition of the Lebesgue measure) shows that the measures $\lambda_{n}$ defined on $N$ by

$$
\lambda_{n}(A):=m_{b} n^{-\mu} \operatorname{card}\left(\frac{1}{n} K \cap A\right)
$$

converge weakly to the Lebesque measure on $N$. Thus,

$$
\frac{S_{n}^{(\mathbf{0})}(M)}{n^{\mu}}=\frac{1}{m_{b}} \lambda_{n}(N) \underset{n \rightarrow \infty}{\longrightarrow} c_{M} .
$$

(c) We consider now the last case, when $\mathbf{k}$ satisfies $\mathbf{k} \in R(M)$, but $\mathbf{k} \neq \mathbf{0}$; in this case, (3.12) holds again, since

$$
S_{n-p}^{(0)}(M) \leq S_{n}^{(\mathbf{k})}(M) \leq S_{n}^{(\mathbf{0})}(M),
$$

where $p$ is the maximal coordinate of some fixed preimage $\mathbf{k}^{\prime}$ of $\mathbf{k}$ (i.e. $\left.\mathbf{k}^{\prime} M=\mathbf{k}\right)$.

Thus, in all cases, we have

$$
\stackrel{S_{n}^{(\mathbf{k})}(M)}{n^{\mu}} c_{M} I^{(\mathbf{k})}\left(M^{*}\right)
$$

Proof of Corollary 1. (1.12) holds when $f^{(e)}$ are trigonometric polynomials (in that case, $\alpha_{M}=\alpha_{M}(0, \ldots, 0)=\mu$, and by Theorem 2 we have $S_{n}(M)=$ $O\left(n^{\mu}\right)$. The same approximation argument used in the proof of Theorem 2 allows us then to extend (1.12) to any functions $f^{(e)} \in L_{p_{e}}$.

\section{ACKNOWLEDGMENT}

We thank R. Bland and R. Fox for useful discussions and unknown referees for simplifying the proofs of case (c) of Lemma 2, and case (b) of Lemma 3. 


\section{REFERENCES}

[AB] F. Avram and L. Brown, A generalized Hölder inequality and a generalized Szëgo theorem, 1987, Preprint.

[A] F. Avram, On bilinear forms in Gaussian random variables and Toeplitz matrices, Probab. Theory Related Fields 79 (1986), 37-45.

[AF] F. Avram and R. Fox, Central limit theorems for sums of Wick products of stationary sequences, Trans. Amer. Math. Soc. 330 (1992), 651-663.

[B] R. Bixby, Matroids and operations research, Preprint.

[BM] P. Breuer and P. Major, Central limit theorem for nonlinear functionals of Gaussian fields, J. Multivariate Analysis 13 (1983), 425-441.

[BP] V. Bryant and H. Perfect, Independence theory in combinatorics, Chapman and Hall, London, 1980.

[F1] R. Fox and M. Taqqu, Non central limit theorems for quadratic forms in random variables having long range dependence, Ann. Probab. 13 (1985), 428-446.

[F2] Central limit theorems in random variables having long range dependence, Probab. Theory Related Fields 74 (1987), 213-240.

[GS] V. Grenander and G. Szëgo, Toeplitz forms and their application, Univ. of California Press, Berkeley, Calif., 1958.

[G1] L. Giraitis and D. Surgailis, C.L.T. theorems and other limit theorems for functionals of Gaussian processes, Z. Wahrsch. Verw. Gebiete 70 (1985), 191-212.

[G2] — Multivariate Appell polynomials and the central limit theorem, Dependence and Independence in Probability and Statistics, (E.. Eberlein and M. Taqqu, eds.), Birkhäuser, 1986.

[K] Y. Katznelson, An introduction to harmonic analysis, Wiley, New York, 1968.

[W] D. Welsh, Matroid theory, Academic Press, New York, 1976.

Department of Mathematics, Northeastern University, Boston, Massachusetts 02115 